\title{
ASSOCIATION BETWEEN TH1 AND TH2 CYTOKINES WITH AXONAL DYSFUNCTION IN SYSTEMIC LUPUS ERYHEMATOSUS
}

\author{
Renan Bazuco Frittoli ${ }^{1}$, Danilo Rodrigues Pereira ${ }^{1}$, Ana Carolina Londe ${ }^{1}$, Mariana Postal ${ }^{1}$, Paula Teixeira Fernandes ${ }^{1}$, Lilian \\ Tereza Lavras Costallat ${ }^{1}$, Roberto Marini ${ }^{1}$, Fernando Cendes ${ }^{1}$, Leticia Rittner ${ }^{1}$, Simone Appenzeller ${ }^{1, \star}$
}

1.Universidade Estadual de Campinas, Campinas (SP), Brazil.

*Corresponding author: appenzellersimone@gmail.com

\section{BACKGROUND}

Neuropsychiatric damage is considered a determinant manifestation of morbidity and mortality in patients with systemic lupus erythematosus (SLE). Abnormalities in the production of Th1 and Th2 cytokines are straight associated with brain metabolic changes. Proton magnetic resonance spectroscopy ( ${ }^{1} \mathrm{H}-\mathrm{MRS}$ ) showed to be an important noninvasive technique to analyze neurological damage. The objective was to determine, longitudinally, the functional brain changes in white matter in SLE and to determine the relationship between the concentrations of neurometabolites with clinical and laboratory findings, drug treatment, Th1 and Th2 cytokines.

\section{METHODS}

We included 147 SLE patients (median age 35 years [SD \pm 11.8$]$ ) from the rheumatology outpatient unit and 80 healthy controls (median age 33 years [SD \pm 9.3$]$ ). Three $1 \mathrm{H}$-MRS exams and blood samples (minimum interval of 1 year) were performed in all individuals. We performed multivoxel $1 \mathrm{H}$-MRS in white matter and signals from $\mathrm{N}$-acetylaspartate (NAA), choline (Cho), creatine ( $\mathrm{Cr}$ ), glutamate and glutamine (GIX) were measured. The serum levels of Th1 (IL-4, IL-6, IL-10) and Th2 (IL-12, TNFa, INF- $\gamma$ ) cytokines were measured using enzyme linked immunosorbent assay (ELISA). Neurological manifestations were analyzed according to the ACR criteria. Anxiety and mood disorders were determined through beck anxiety (BAI) and beck depression inventory (BDI). Systemic lupus erythematosus patients were further assessed for clinical and laboratory SLE manifestations, disease activity (SLE Disease Activity Index [SLEDAI]), damage (Systemic Lupus International Collaborating Clinics/American College of Rheumatology Damage Index [SDI]). Data were compared by nonparametric tests.

\section{RESULTS}

$\mathrm{N}$-acetylaspartate and choline ratio ( $\mathrm{NAA} / \mathrm{Cr}$ ) remained significantly reduced in patients and was associated with the presence of cumulative damage $(p=0.029)$, psychosis $(p=0.027)$, seizure $(p=0.048)$ and inversely correlated with corticosteroid dose ( $r s=-0.17 ; p=0.038$ ). The $\mathrm{Cho} / \mathrm{Cr}$ ratio revealed a significant increase in patients with neuropsychiatric damage, associated with depression ( $p=0.019)$, headache $(p=0.005)$, cognitive impairment $(p=0.04)$ and anticardiolipin antibody $(p=0.019)$. Significant fluctuation was detected in all cytokines in the Th2 group (IL-4 [ $p=0.001]$, IL-6 [ $p=0.004]$ and IL-10 [p=0.0002]). The IL-6 and IL-10 remained significantly increased in SLE patients when compared to controls. There was an inverse correlation between NAA/ $\mathrm{Cr}$ and levels of INFY $(\mathrm{rs}=-0.26, \mathrm{p}=012)$ and $\mathrm{IL}-6(\mathrm{rs}=-0.25, \mathrm{p}=0.017)$.

\section{CONCLUSION}

Decrease of NAA/Cr ratio was more frequent in SLE patients and was associated with the presence of psychosis, seizure and inversely correlated with corticosteroid dose, INF- and IL-6. The Cho/Cr ratio was higher in patients with neuropsychiatric manifestations. Serum levels of cytokines IL-6 and IL-10 were increased in patients and IL-10 was associated with disease activity. Cytokines are associated with axonal dysfunction and NAA and Cho can be used as biomarkers of neuropsychiatric lupus.

\section{KEYWORDS}

Proton magnetic resonance spectroscopy, Systemic lupus erythematosus, White matter, Cytokines. 